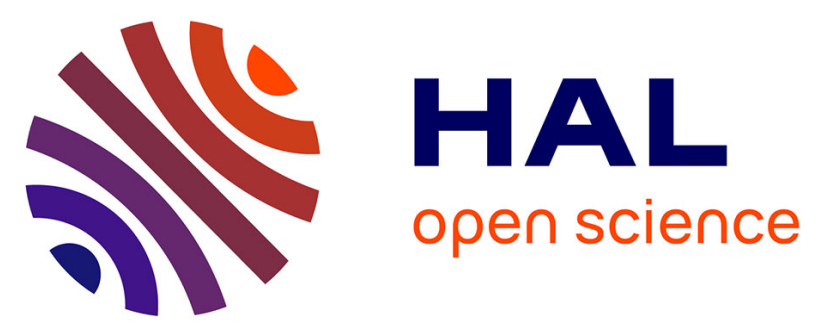

\title{
Performance improvement of plasmonic sensors using a combination of AC electrokinetic effects for (bio)target capture
}

Quentin Avenas, Julien Moreau, Marion Costella, Arbi Maalaoui, Abdelkader Souifi, Paul Charette, Julien Marchalot, Marie Frénéa-Robin, Michael Canva

\section{To cite this version:}

Quentin Avenas, Julien Moreau, Marion Costella, Arbi Maalaoui, Abdelkader Souifi, et al.. Performance improvement of plasmonic sensors using a combination of $\mathrm{AC}$ electrokinetic effects for (bio)target capture. Electrophoresis, 2019, 40, pp.1426 - 1435. 10.1002/elps.201800436 . hal02073261

\section{HAL Id: hal-02073261 https://hal.science/hal-02073261}

Submitted on 17 Dec 2020

HAL is a multi-disciplinary open access archive for the deposit and dissemination of scientific research documents, whether they are published or not. The documents may come from teaching and research institutions in France or abroad, or from public or private research centers.
L'archive ouverte pluridisciplinaire HAL, est destinée au dépôt et à la diffusion de documents scientifiques de niveau recherche, publiés ou non, émanant des établissements d'enseignement et de recherche français ou étrangers, des laboratoires publics ou privés. 


\title{
$<\mathrm{PE}-\mathrm{AT}>$ Performance improvement of plasmonic sensors
}

\section{using a combination of AC electrokinetic effects}

\author{
for (bio)target capture
}

\author{
Quentin Avenas ${ }^{1,2}$, Julien Moreau 4 , Marion Costella ${ }^{1,3}$, Arbi Maalaoui ${ }^{1,3}$, \\ Abdelkader Souifi ${ }^{2}$, Paul Charette ${ }^{1}$, Julien Marchalot ${ }^{3}$, Marie Frénéa-Robin ${ }^{3}$ and \\ Michael Canva ${ }^{1,4}$ \\ ${ }^{1}$ Laboratoire Nanotechnologies et Nanosystèmes, LN2 ; CNRS - Université de Sherbrooke - INSA Lyon ; 3IT, \\ Sherbrooke, Canada \\ ${ }^{2}$ Institut des Nanotechnologies de Lyon ; CNRS - INSA Lyon - Université Claude Bernard Lyon 1;
}

Villeurbanne, France

${ }^{3}$ AMPERE ; CNRS - Université de Lyon - École Centrale Lyon - INSA Lyon - Université Claude Bernard Lyon 1 ; F69130, Ecully, France

${ }^{4}$ Laboratoire Charles Fabry ; CNRS - Institut d'Optique Graduate School, Université Paris Saclay ; Palaiseau, France

Corresponding author: Quentin Avenas

Title: PhD Student

Full postal address: 111 rue Cuvier, 69006 Lyon

E-mail address: quentin.avenas@usherbrooke.ca

(iDhttps://orcid.org/0000-0002-1006-6412

List of abbreviations:

ACEO: Alternating current electroosmosis

DEP: Dielectrophoresis

Received: 10 15, 2018; Revised: 01 15, 2019; Accepted: 02 04, 2019

This article has been accepted for publication and undergone full peer review but has not been through the copyediting, typesetting, pagination and proofreading process, which may lead to differences between this version and the Version of Record. Please cite this article as doi: 10.1002/elps.201800436.

This article is protected by copyright. All rights reserved. 
ETE: Electrothermal effect

IDE: Interdigitated electrodes

RIU: Refractive index unit

ROI: Region of interest

SPR: Surface plasmon resonance

SPRI: Surface plasmon resonance imaging

TE: Transverse electric

TM: Transverse magnetic

UV: Ultraviolet

Keywords: Biosensor, Dielectrophoresis; Electroosmosis; Surface plasmon resonance.

\section{Abstract}

Analytes concentration techniques are being developed with the appealing expectation to boost the performance of biosensors. One promising method lies in the use of electrokinetic forces. We present hereafter a new design for a microstructured plasmonic sensor which is obtained by conventional microfabrication techniques, and which can easily be adapted on a classical surface plasmon resonance imaging (SPRI) system without further significant modification. Dielectrophoretic trapping and electro-osmotic displacement of the targets in the scanned fluid are performed through interdigitated $200 \mu \mathrm{m}$ wide gold electrodes that also act as the SPR-sensing substrate. We demonstrate the efficiency of our device's collection capabilities for objects of different sizes (200 $\mathrm{nm}$ and $1 \mu \mathrm{m}$ PS beads, as well as $5-10 \mu \mathrm{m}$ yeast cells). SPRI is relevant for the spatial analysis of the mass accumulation at the electrode surface. We demonstrate that our device overcomes the diffusion limit encountered in classical SPR sensors thanks to rapid collection capabilities $1<1$ minute) and we show a consequent improvement of the detection limit, by a factor greater than 300. This study of an original device combining SPRI and electrokinetic forces paves the way to the development of fully integrated active plasmonic sensors with direct applications in life sciences, electrochemistry, environmental monitoring and agri-food industry.

Color online: See article online to view Figs. 1-5 in color.

Additional supporting information may be found in the online version of this article at the publisher's web-site.

\section{<PE-FRONTEND>}

\section{Introduction}

\subsection{SPRI}

Surface Plasmon Resonance (SPR) is a powerful detection technique for analyzing biomolecular interactions [1]-[3] with applications in various domains such as life sciences, electrochemistry or 
food and environment safety. SPR sensors allow for label-free, real-time, quantitative and sensitive detection and/or analysis [4] of intermolecular events. They are usually used in the Kretschmann configuration, as described on Figure 1.a): the metal film is illuminated through a prism by an incident beam that is then reflected except for a particular angle and wavelength where light is absorbed by the electrons at the metal-dielectric interface. These resonance angle and wavelength depend on the optical properties of the materials near the interface. Biomolecular events at the surface (e.g. target/probe interaction as illustrated on Figure 1.a)) locally change the refractive index and are thus detected by tracking the coupling resonance angle (or wavelength) shifts. In a complementary manner SPR Imaging (SPRI) usually consists in the measurement of reflectivity variations at a fixed angle and wavelength. This technique provides spatial information [5],[6] and led to the development of high-throughput SPR biochips [7]-[10]. The high interest in this technology has led different research groups to reach the maximal theoretical resolution limit in the last decade [11]. However, as a surface-based sensing technique, it can only detect molecules if they are near the surface (typically, less than $200 \mathrm{~nm}$ away). Thus, the diffusion limit is the main obstacle to performance enhancement of all surface-based sensors today [12], and particularly SPRI biochips.

Figure_1

\subsection{Electrohydrodynamic (EHD) forces}

Microfluidic transport strategies have been studied since the last two decades in order to overcome the diffusion limit [13]. Amongst all the existing techniques, electrokinetic effects are particularly interesting because they are applicable to a wide range of target sizes and types. They also can be induced within devices of various materials and geometries [14], depending on the objective. Thus, they could easily be adapted to the structure of a SPRI sensor using the gold sensing surface as an electrode and thereby bring an answer to the problem of the diffusion limit.

Electrokinetic effects such as electrophoresis, dielectrophoresis (DEP), alternating current electro-osmosis (ACEO) and electro-thermal effect (ETE) have been widely studied [15]-[19] and enable different ways of breaking this limit. Since electrophoresis is conventionally carried out using a direct current field, undesirable effects like alteration of the gold surface may affect the monitored signal. Hence we chose to use AC electrokinetic effects and to implement them on an SPRI sensor.

Pioneering results on sensor enhancement by combined EHD/DEP were reported from 2003 by Hughes' group [20]-[23]. Coupling of electrokinetic actuation and evanescent light sensing on the same device was demonstrated for the first time in 2003 by the same group [24] and AC electric field enhanced nanoplasmonic detection has been reported by two different groups [25],[26] more recently. In this paper, we demonstrate that SPRI can be used in conjunction with AC electrokinetic effects not only to improve the sensor response but also to analyse the respective contributions of DEP and ACEO effects.

\section{Materials and methods}

\subsection{Design and fabrication of the device}

Our device consists of coplanar gold interdigitated electrodes (IDE) on a microscope glass slide, as shown on Figure 1.c). Rectangular IDE enable massive parallel manipulation of objects as they can easily be arranged in large arrays [27]. Additionally, their simple fabrication process

This article is protected by copyright. All rights reserved. 
involves a single photolithography step. Moreover, their symmetrical geometry enables a theoretical study of the device that fits well with the experimental results, as demonstrated later. Lastly, the fact that the electrodes are integrated on the same substrate makes it easily adaptable to a classical SPRI system, in which they can also be used as sensing areas.

Electrodes are $200 \mu \mathrm{m}$ wide and separated with a $150 \mu \mathrm{m}$ gap. These dimensions enable easy and rapid fabrication of the biochip. A large gold electrode is also present $1 \mathrm{~mm}$ away from the IDE, whose purpose is to provide a comparison with a classical SPRI sensor during experiments. It is not connected to the electrical circuit and it will be referred to as the "control electrode".

The fabrication process is straightforward: the substrate, a classical BK7 glass microscope slide, was first cleaned with acetone, ethanol and water. Then an adhesion layer of Ti $(3 \mathrm{~nm})$ and the transducing layer of $\mathrm{Au}(47 \mathrm{~nm})$ were evaporated onto the substrate. The samples were then spincoated with AZ5214 (MicroChem) and insulated with UV (within an UV-Kub) before the photoresist development using AZ726MIF developer. The principal advantage of the fabrication technique lies in the use of an acetate photomask during this step, which reduces the cost of each new design while suiting our resolution requirements. Finally, the metal layers were etched with Gold etchant and Titanium etchant, the photoresist was stripped with acetone and the samples were cleaned again with acetone, ethanol and DI water.

\subsection{Experimental protocol}

\subsubsection{Solution preparation}

Aqueous solutions containing different analytes were studied. Polybead ${ }^{\circledR}$ Amino Microspheres of $200 \mathrm{~nm}$ and $1 \mu \mathrm{m}$ diameter were purchased from Polysciences. Each beads solution was prepared by diluting the stock suspension with ultrapure water whose conductivity before the dilutions was measured to be $5.5 \times 10^{-6} \mathrm{~S} / \mathrm{m}$. The initial concentration of the commercial solution of $200 \mathrm{~nm}$ beads was $5.68 \times 10^{12}$ particles $/ \mathrm{ml}$, that of the $1 \mu \mathrm{m}$ beads solution was $1.13 \times 10^{12}$ particles $/ \mathrm{mL}$. Both types of beads have a positive Clausius-Mossotti (CM) factor in pure water at the considered frequencies (see Figure 4 and section 3.3 about the MyDEP calculations) and are therefore expected to experience positive dielectrophoresis. Solutions of beads were diluted 1000 times for the trapping experiments and spatial study. Several dilutions of the $200 \mathrm{~nm}$ beads solution, detailed in Figure 5.b), were made for the study of detection limit enhancement. Baker's yeast cells (saccharomyces cerevisiae, purchased from Vahine ${ }^{\mathrm{TM}}$ ) were used as biological model objects. They were rehydrated in water and resuspended at a concentration evaluated to $4.3 \times 10^{4}$ cells $/ \mathrm{ml}$ with a Malassez counting cell.

For the calculation of the $\mathrm{CM}$ factor, a spherical model of concentric layers was adopted. We used a radius of $3.5 \mu \mathrm{m}$ and considered the cell membrane and the cell wall to be respectively $8 \mathrm{~nm}$ and $220 \mathrm{~nm}$ thick. Input values for the electrical conductivity of the cytoplasm, the cell membrane and the cell wall were respectively $0.3 \mathrm{~S} / \mathrm{m}, 25 \mu \mathrm{S} / \mathrm{m}$ and $24 \mathrm{~ms} / \mathrm{m}$. The dielectric constants of these compartments were respectively set to 50, 6 and 60 (default values extracted from MyDEP database, and originated from [28]). 


\subsubsection{Experimental workflow}

Our device was first mounted on a BK7 glass prism in the Kretschmann configuration. Oil was used to optimize the optical contact between the SPRI chip and the prism. A $90 \mu \mathrm{m}$ thick Mylar ${ }^{\circledast}$ seal was placed onto the chip before the prism/chip/Mylar ${ }^{\circledR}$ system was clamped against the wall of the experimental bench. This supporting wall has two holes through which medium enters and exits the fluidic cell. A photography of this system is provided in the Supporting Information (Figure S1). Fluid flow was induced through the fluid circuit thanks to a peristaltic pump (Gilson, Minipuls 3 ).

At the beginning of each experiment, pure water was injected in the fluidic cell and an angular scan was performed to determine the working angle at the chosen wavelength $(740 \mathrm{~nm})$. The camera (Hamamatsu, C11440), motors (used to move the camera and the light source) and thermal regulation of the fluidic cell were controlled with a LabVIEW program. Before the beginning of each electrokinetic experiment, a stable baseline was acquired and a calibration step was performed with a glycerol/water solution, equivalent to a variation of $1 \times 10^{-3}$ RIU (Refractive Index Units) [7]. Then the function generator (Agilent, 33120A) was operated manually to apply a sinusoidal signal. The application of an electric potential at a low frequency on the gold sensing surface can affect the SPR signal even in pure water, due to oxidation of the metal [29],[30] or changes in the repartition of charges at the metal/dielectric interface [31]. However, we verified experimentally that with the electrical parameters we used (frequency greater than $500 \mathrm{~Hz}$, voltage lower than $10 \mathrm{Vpp}$ ) it had no influence on our SPR measurements, which is consistent with the literature [29].

Two different processes were used for particle collection under AC electrokinetic effects. For the spatial study, the experiments consisted in successive identical sequences: the sample was injected, the pump was switched off and then the function generator was switched on after a short relaxation step. There was therefore no flow during the mass transport step. After particle collection, the function generator was switched off and fresh medium was injected in the fluidic cell before the beginning of the next sequence. The protocol used for determining the limit of detection was different, as the electric field was applied during the injection in order to maximize the quantity of trapped objects.

\subsubsection{Imaging methods}

The SPRI system was described in a previous article [32] and is schematized on Figure 1.c). The fabricated device is mounted on a BK7 glass prism and clamped against the fluidic cell. Electrode contact pads are electrically connected to a function generator thanks to alligator clips.

Images in TE and TM polarizations were collected during the experiment at a framerate of approximately 1 image/s. Intensity measurements on different regions of interest (ROI) were then extracted using ImageJ software, and calculations were made afterwards in order to convert them into RIU variations, based on the calibration step [7]. Intensity measurements were also conducted on the reference electrode, which is not connected to the electrical circuit, for comparison with the signal that would be obtained with a classical SPR biochip. Moreover, diffusive effects induced a second phase of slow signal growth after the rapid trapping phase. In this study we focused on the rapid collection of particles. Thus, for the last part of this study, the signal was measured after a fixed time of $30 \mathrm{~s}$ which was the same for each measurement, therefore ensuring their comparability. 
Optical microscopy observations (using a $20 \times$ objective) were made with an Axiovert 40 Zeiss microscope (used in bright field mode) and an Eclipse LV150 Nikon microscope (used in dark field mode).

\section{Results and discussion}

\subsection{Collection of different particles}

Collection of all the different particles ( $200 \mathrm{~nm}$ beads, $1 \mu \mathrm{m}$ beads and yeast cells) was accomplished and observed by SPRI. $1 \mu \mathrm{m}$ beads were trapped by applying a sinusoidal signal at 20 Vpp and $5 \mathrm{kHz}$ (Figure 2.b)). Images of the collection of these beads were also acquired by optical microscopy, as shown on Figure 2.a). SPRI shows precise localization of the beads, forming symmetrical bands over the electrodes whereas optical microscopy shows a more homogeneous repartition over the electrode surface. While the same electrical parameters were used during those two experiments, the observable result is different because optical microscopy only allows for seeing volume effects, whereas SPRI allows for sensing what happens directly at the surface.

Figure_2

The reasons for this collection pattern in bands have been explained previously [14]: at this frequency, beads are subjected to positive DEP (the crossover frequency is approximately $2 \mathrm{MHz}$, see Figure 4) and ACEO simultaneously. Positive DEP exerts a force attracting beads to the electrode edges while ACEO induced vortices that drag them towards the centre of the electrodes. Combined action of these two forces is summarized on Figure 1.b). An equilibrium between both forces is attained at this specific frequency, and the beads form bands at the point where the sum of the forces is null. ETE's influence on the collection pattern is negligible at this conductivity [14].

A similar trapping behavior was observed with the $200 \mathrm{~nm}$ beads subjected to identical field conditions, as observed by SPRI. The collection pattern dynamics (formation of bands that progressively shift towards the electrode center) is clearly observable and is shown on Figure 2.c). It is worth noting that switching of the electric field immediately causes the beads to detach from the electrode surface and become unobservable. This could not have been observed by optical microscopy and this highlights one the reason of the relevance of SPRI for biosensing: it enables the detection of sub-micron objects.

SPRI observations of yeast cell collection (Figure 2.d) and 2.e)) show that our system is also efficient for trapping and sensing biological objects. Their different dielectric properties and size distribution induce a collection pattern different from that of the beads as well as different optimal electrical parameters for collection (the frequency was $10 \mathrm{kHz}$ in this case) as observed elsewhere in the literature [21].

\subsection{SPR as a tool for the study of AC electrokinetic effects: a study of near-surface dynamics}

As shown on Figure 2, our SPRI device is capable of providing spatial information about nearsurface dynamics of the particles which is otherwise unavailable. Being able to analyze these phenomena and study the mass transport dynamics at a surface level is important when working on problematics such as active functionalization, for which it is essential to know where the particles are collected. 
We performed a series of experiments at three different frequencies $(500 \mathrm{~Hz}, 1 \mathrm{kHz}$ and 2 $\mathrm{kHz}$ ) and amplitudes in the 2-20 Vpp range with a solution of $200 \mathrm{~nm}$ beads diluted 1000 times. Intensity measurements were then extracted from a sequence of identical contiguous ROI across the width of one electrode. Calculations were performed to evaluate the equivalent RIU variations, which are displayed as a function of the amplitude and the position on the electrode in a 3D representation shown on Figure 3.

Figure_3

At $1 \mathrm{kHz}$ and $2 \mathrm{kHz}$, the equilibrium position of the beads shifts when the amplitude changes. At $2 \mathrm{Vpp}$ the resulting forces are not strong enough to observe the effect of DEP and ACEO. Collection of the beads begins to be observable starting from $6 \mathrm{Vpp}$. At this amplitude, at $t=30 \mathrm{~s}$, beads are trapped near the edges of the electrodes. Their position at this time shifts progressively towards the center of the electrode when the amplitude is increased. This shift is faster at $1 \mathrm{kHz}$, and the displacement of the DEP/ACEO equilibrium is not observed at all when the frequency is lowered to $500 \mathrm{~Hz}$.

In order to understand this behaviour, we studied theoretically the influence of both forces. Equation 1 describes the expression of the dielectrophoretic force for a spherical particle with a radius $r$, where $E_{\text {eff }}$ is the RMS value of the electric field, $\varepsilon_{p}^{*}$ is the complex permittivity of the particle and $\varepsilon_{m}^{*}$ that of the medium. The DEP force is strongly influenced by the Clausius-Mossotti factor CM, given by Equation 2: if its real part is positive, DEP will be positive (i.e. the force acts in the gradient direction and therefore drives the particle towards areas of maximum field intensity). On the contrary, if $\operatorname{Re}[C M]$ is negative, DEP is negative, which means that the force will move the particle against the gradient.

$$
\begin{gathered}
<F_{D E P}>=2 \pi \varepsilon_{m} r^{3} \operatorname{Re}[C M] \nabla E_{\text {eff }}^{2} \\
C M=\frac{\varepsilon_{p}^{*}-\varepsilon_{m}^{*}}{\varepsilon_{p}^{*}+2 \varepsilon_{m}^{*}}
\end{gathered}
$$

We calculated the Clausius-Mossotti factor for each studied type of particle (beads of both sizes and yeast cells) using the MyDEP software [33] and experimental parameters obtained previously (see Supporting Information). Results are displayed as a function of the frequency on Figure 4.c). For the 3 chosen frequencies $(500 \mathrm{~Hz}, 1 \mathrm{kHz}$ and $2 \mathrm{kHz}$ ), the Clausius-Mossotti factor is positive and constant, meaning that the particles are attracted to the electrode edges in an identical way in each of the three cases.

$$
<u_{x}>=\frac{1}{8} \frac{\varepsilon_{m} \varphi_{0}^{2} \Omega^{2}}{\eta x\left(1+\Omega^{2}\right)^{2}}
$$

The electroosmotic velocity field in the fluid on the electrodes was calculated according to the theoretical explanations formerly detailed by Morgan and Green [35]. Electroosmotic velocity at a distance $x$ from the electrode edge is given by Equation 3, where $\varphi_{0}$ is the applied potential, $\eta$ is the dynamic viscosity of the fluid and $\Omega=1 / 2 \pi \kappa x \omega\left(\varepsilon_{m} / \sigma_{m}\right)$ is a non-dimensional frequency depending on the Debye length $1 / \kappa$, the medium conductivity $\sigma_{m}$ and the angular frequency of the electric field $\omega$. 
The electroosmotic velocity of the fluid on the electrode surface, at a distance $x=50 \mu \mathrm{m}$ from the electrode edge, is displayed on Figure 4.a), clearly showing different behaviours for the 3 different chosen frequencies. The velocity is almost at its maximum at $500 \mathrm{~Hz}$, intermediate at $1 \mathrm{kHz}$ and near to a minimum at $2 \mathrm{kHz}$. This explains the shape of the 3D graphs on Figure 3: at $500 \mathrm{~Hz}$ where the ACEO velocity is maximal, beads are dragged towards the electrode center even at the lowest amplitude whereas for higher frequencies, DEP influence is stronger for lower amplitudes. Additionally, the shift towards the center when increasing the amplitude is faster at $1 \mathrm{kHz}$ than at 2 $\mathrm{kHz}$ because the electroosmotic velocity is higher.

Figure_4

Distribution profiles of the beads over the electrode width were extracted from the experimental results for each frequency and a given amplitude of $10 \mathrm{Vpp}$ (see Figure 4.b). They clearly show the shift of the collection site of the beads when changing the frequency. The displacement being towards the electrode center, these findings are consistent with the theoretical results and illustrate the dominance of ACEO over DEP at lower frequencies.

Furthermore, this DEP/ACEO equilibrium is strongly dependent on the nature and size of the analytes. In that respect, SPRI observations of the trapping of $1 \mu \mathrm{m}$ beads (see Error! Reference source not found..b)) show that bigger objects are more attracted to the electrode edges, which is why the bands of $1 \mu \mathrm{m}$ beads are closer to the electrode edge after $30 \mathrm{~s}$ of applying the voltage compared with the bands of $200 \mathrm{~nm}$ beads. The size dependence of the collection dynamics is understandable through the equations of both $\mathrm{AC}$ electrokinetic effects. Electroosmotic flow induces a drag force on the beads which is proportional to their radius whereas the DEP force, which is still positive as the $\mathrm{CM}$ factor on Figure 4.c) shows it, is proportional to the volume (i.e. to $r^{3}$ ). Thus, for a bigger object and with every other parameter fixed, DEP grows faster so the collection bands are shifted to the edge (see supplementary information, Figure S2).

Yeast cells (saccharomyces cerevisiae) are different in nature and size. The DEP force that applies on them is still positive for the considered frequency $(10 \mathrm{kHz})$, as indicated by the $\mathrm{CM}$ factor (see Figure 4.c)) whereas the electroosmotic velocity is lower at this frequency. If some yeast cells can be seen individually on the electrode surface after applying the voltage, the occurrence of circular irregularities on the electrode edges (Figure 2.e)) indicates a probable predominant attraction of the yeast cells towards the edges, which was later confirmed thanks to optical microscopy observations (Figure 2.f)). Thus, it seems that the DEP force is predominant in this case, which is consistent with our previous explanations about the influence of the target size on the DEP/ACEO equilibrium. The fact that yeast cells are trapped on the edges could be problematic for SPRI observations, but other electrode configurations, such as face-to-face electrodes (paper to be published), solve this easily.

These results prove that the position of the analytes on the electrodes can be electrically controlled and that the collection patterns can be predicted using theoretical tools. Besides, this initial study illustrates the fact that SPRI is particularly suitable for the study of near-surface dynamics when mass transport at a micron scale is involved. 


\subsection{Detection limit enhancement}

Knowing that we were able to collect beads on the electrodes, our next objective was to quantify the performances of our device. Being able to predict the exact position of the beads allows for choosing precisely the region over which the signal is quantified, in order to get the maximal sensitivity: in this case, beads are collected at the center of the electrodes and thus, we measured the signal in this region of interest (ROI). We checked that switching on the electric field had no influence on the measured signal when no beads were present in the solution: the signal remained constant while applying the voltage ( $2 \mathrm{kHz}, 20 \mathrm{Vpp}$ ) on ultrapure water only. This negative control is plotted on Figure 5.a) for comparison.

\section{Figure_5}

In order to show the signal enhancement capabilities of our device and to explain how the signal variations are calculated, an example of the kinetics of the signal during collection of a solution diluted 1000 times is shown on Figure 5.a). The signal for the microstructured sensor was extracted at the electrode center. The signal corresponding to a classical SPR sensor was extracted from an ROI of the same size on the reference electrode. After injection of the beads and a short relaxation period, the function generator was switched on, inducing mass transport of the particles. This generated a huge and rapid rise in the measured signal. The function generator was then switched off, causing the beads to detach from the gold surface and the signal to come back to its baseline. As shown on Figure 5.a), the signal variation of our enhanced device is much bigger than that of a classical SPR sensor.

In order to determine the limit of detection of our device, several dilutions of the $200 \mathrm{~nm}$ beads solution were made, issuing solutions diluted 1000, 3000, 10000, 30 000, 100000 and 300000 times. The different solutions were studied successively on the same sample and with the same electrical parameters $(2 \mathrm{kHz}, 20 \mathrm{Vpp})$. We started with the most diluted solution and went gradually towards the most concentrated one, in order to prevent a hysteresis effect in the signal that could be due to bead accumulation on the surface.

During this series of experiments showing the enhancement of the limit of detection, there was no relaxation time: the function generator was switched on before bead injection. We verified experimentally that this process increased the quantity of trapped objects, affecting the signal variations in the same way. As explained earlier, we measured the signal variation at a fixed time of $30 \mathrm{~s}$ after the beginning of the particles collection because we were interested in the first-order dynamics of the trapping process.

The white noise was calculated on every ROI separately. The limit of detection is defined as the triple of this quantity. Thus, every signal variation inferior to this quantity is not meaningful. The limit of detection is the concentration which marks the boundary between the range where the sensor is efficient (providing signal variations greater than the detection threshold) and the range where it is not.

Figure 5.b) shows the measured signal variations for every dilution, enabling the comparison between our enhanced sensor and a classical SPR sensor. The calculated noise is plotted as a line for convenience. On a classical SPR sensor, every solution diluted more than 3000 times induces a signal variation which is inferior to the noise limit, while the enhanced sensor gives a signal for every 
solution, including the most diluted one with a 1000000 dilution factor). Hence, we can conclude that our device enables the enhancement of the limit of detection by a factor greater than $\mathbf{3 0 0}$ in the given measurement time interval. This experiment was repeated multiple times and similar results were obtained, issuing the same (or greater) enhancement factor.

\section{Conclusion and perspectives}

We presented a new design for a SPRI sensor capable of operating AC electrokinetic effects in order to trap the target analytes onto its sensing surface. We demonstrated the efficiency of its collection capabilities for objects of different sizes ( $200 \mathrm{~nm}$ and $1 \mu \mathrm{m}$ PS beads, yeast cells of about $7.5 \mu \mathrm{m})$. The observed mass transport dynamics were understood and theoretically explained with an excellent consistency with the experimental results. In this regard, SPR appears to be a particularly well-suited tool for the study of AC electrokinetic effects dynamics near the surface of the electrodes. Finally, we demonstrated that our device achieves its goal of overcoming the diffusion limit, by showing that the limit of detection could be enhanced by more than two orders of magnitude.

Aside from highlighting one more achievement in the field of SPRI sensing, this work paves the way to the development of a new generation of active plasmonic biosensors, capable of controlling mass transport to enhance their performance. The fully integrated approach presented here has the advantage of allowing for excellent compatibility of the biochip component with already existing SPR systems and thus for rapid development, commercialization and routine use whenever needed devices. Furthermore, other configurations, such as face-to-face electrodes, or other substrate modifications, such as nanostructuration, also have their own advantages and are currently under further studies.

\section{Acknowledgements}

LN2 is an international laboratory (Unité Mixte Internationale UMI 3463) jointly managed by French CNRS and the Université de Sherbrooke as well as the Universite de Lyon (INSA de Lyon, ECL, CPE Lyon) and the Université Grenoble Alpes (UGA). Quentin Avenas acknowledges support from the French doctoral scholarship as well as MITACS and the IDEX Lyon program. He also thanks the Laboratoire Charles Fabry and the Laboratoire Ampère for providing access to their equipment.

The authors have declared no conflict of interest.

\section{REFERENCES}

[1] Homola, J., Piliarik, M., in Surface Plasmon Resonance Based Sensors (Ed.: Homola, J.), Springer, Berlin, 2006.

[2] Campbell, C., Kim, G., Biomaterials 2007, 28, 2380-2392.

[3] Moreau, J., Cloarec, J.-P., Charette, P. G., Barbillon, G., Canva, M., In Handbook of Enhanced Spectroscopy, Taylor \& Francis Group, New York, 2015. 
[4] Moreau, J., Cloarec, J.-P., Charette, P., Goossens, M., Canva, M., Vo-Dinh, T., In Biomedical Photonics Handbook, Second Edition: Biomedical Diagnostics (Ed.: Vo-Dinh, T.), CRC Press, Boca Raton, 2014, pp. 199-264.

[5] Rothenhäusler, B., Knoll, W., Nature 1998, 615-617.

[6] Brockman, J. M., Nelson, B. P., Corn, R. M., Annu. Rev. Phys. Chem. 2000, 51, 41-63.

[7] Bardin, F., Bellemain, A., Roger, G., Canva, M., Biosens. Bioelectron. 2009, 24, 2100-2105.

[8] Piliarik, M., Párová, L., Homola, J., Biosens. Bioelectron. 2009, 24, 1399-1404.

[9] Hottin, J., Moreau, J., Roger, G., Spadavecchia, J., Millot, M.-C., Goossens, M., Canva, M., Plasmonics 2007, 2, 201-215.

[10] Boozer, C., Kim, G., Cong, S., Guan, H., Londergan, T., Curr. Opin. Biotechnol. 2006, 17, 400-405.

[11] Piliarik, M., Homola, J., Optics Express 2009, 17, 16505-16517.

[12] Sheehan, P. E., Whitman, L. J., Nano Lett. 2005, 5, 803-807.

[13] Lee, C.-Y., Chang, C.-L., Wang, Y.-N., Fu, L.-M., Int. J. Mol. Sci. 2011, 12, 3263-3287.

[14] Oh, J., Hart, R., Capurro, J., Noh, H. (Moses), Lab Chip 2009, 9, 62-78.

[15] Green, N. G., Ramos, A., González, A., Morgan, H., Castellanos, A., Phys. Rev. E 2000, 61, 4011.

[16] González, A., Ramos, A., Green, N. G., Castellanos, A., Morgan, H., Phys. Rev. E 2000, 61, 4019.

[17] Green, N. G., Ramos, A., González, A., Morgan, H., Castellanos, A., Phys. Rev. E 2002, 66.

[18] Bazant, M. Z., Squires, T. M., Phys. Rev. Lett. 2004, 92.

[19] Ren, Y., Song, C., Liu, W., Jiang, T., Song, J., Wu, Q., Jiang, H., Electrophoresis 2018; doi: 10.1002/elps.201800325.

[20] Hoettges, K. F., McDonnell, M. B., Hughes, M. P., J. Phys. D: Appl. Phys. 2003, 36, L101.

[21] Hübner, Y., Hoettges, K. F., McDonnell, M. B., Carter, M. J., Hughes, M. P., Int. J. Nanomed. 2007, 2, 427.

[22] Fatoyinbo, H. O., Hoettges, K. F., Reddy, S. M., Hughes, M. P., Biosens. Bioelectron.2007, 23, 225-232.

[23] Mohtar, M. N., Hoettges, K. F., Hughes, M. P., Electrophoresis 2014, 35, 345-351.

[24] Hoettges, K. F., Hughes, M. P., Cotton, A., Hopkins, N. A., McDonnell, M. B., IEEE Eng. Med. Biol. Mag. 2003, 22, 68-74.

[25] Barik, A., Otto, L. M., Yoo, D., Jose, J., Johnson, T. W., Oh, S.-H., Nano Lett. 2014, 14, 2006-2012.

[26] Kuroda, C., lizuka, R., Ohki, Y., Fujimaki, M., Japan. J. Appl. Phys. 2018, 57, 057001.

[27] Menad, S., El-Gaddar, A., Haddour, N., Toru, S., Brun, M., Buret, F., Frenea-Robin, M., Langmuir 2014, 30, 5686-5693.

[28] Talary, M. S., Burt, J. P. H., Tame, J. A., Pethig, R., J. Phys. D: Appl. Phys. 1996, 29, 2198-2203.

[29] Lioubimov, V., Kolomenskii, A., Mershin, A., Nanopoulos, D. V., Schuessler, H. A., Appl. Optics 2004, 43, 3426-3432.

[30] Huang, Y., Pitter, M. C., Somekh, M. G., Zhang, W., Xie, W., Zhang, H., Wang, H., Fang, S., Sci. China Phys. Mech. Astron. 2013, 56, 1495-1503.

[31] Lopatynskyi, A. M., Lopatynska, O. G., Poperenko, L. V., Chegel, V. I., Guiver, M. D., Semiconductor Physics Quantum Electronics and Optoelectronics, National Academy of Sciences of Ukraine, Kyiv 2008.

[32] Sereda, A., Moreau, J., Canva, M., Maillart, E., Biosens. Bioelectron. 2014, 54, 175-180.

[33] Cottet, J., Fabregue, O., Berger, C., Buret, F., Renaud, P., Frénéa-Robin, M., Biophys. J. 2019, 116, 12-18.

[34] Menad, S., Assemblage permanent de micro-objets par diélectrophorèse associée à une méthode de couplage covalent, PhD Thesis, Autre, Ecole Centrale de Lyon 2014.

[35] Morgan, H., Green, N. G., AC electrokinetics: colloids and nanoparticles, Microtechnologies and microsystems series, Research Studies Press, Baldock, Philadelphia 2003. 
Figure 2
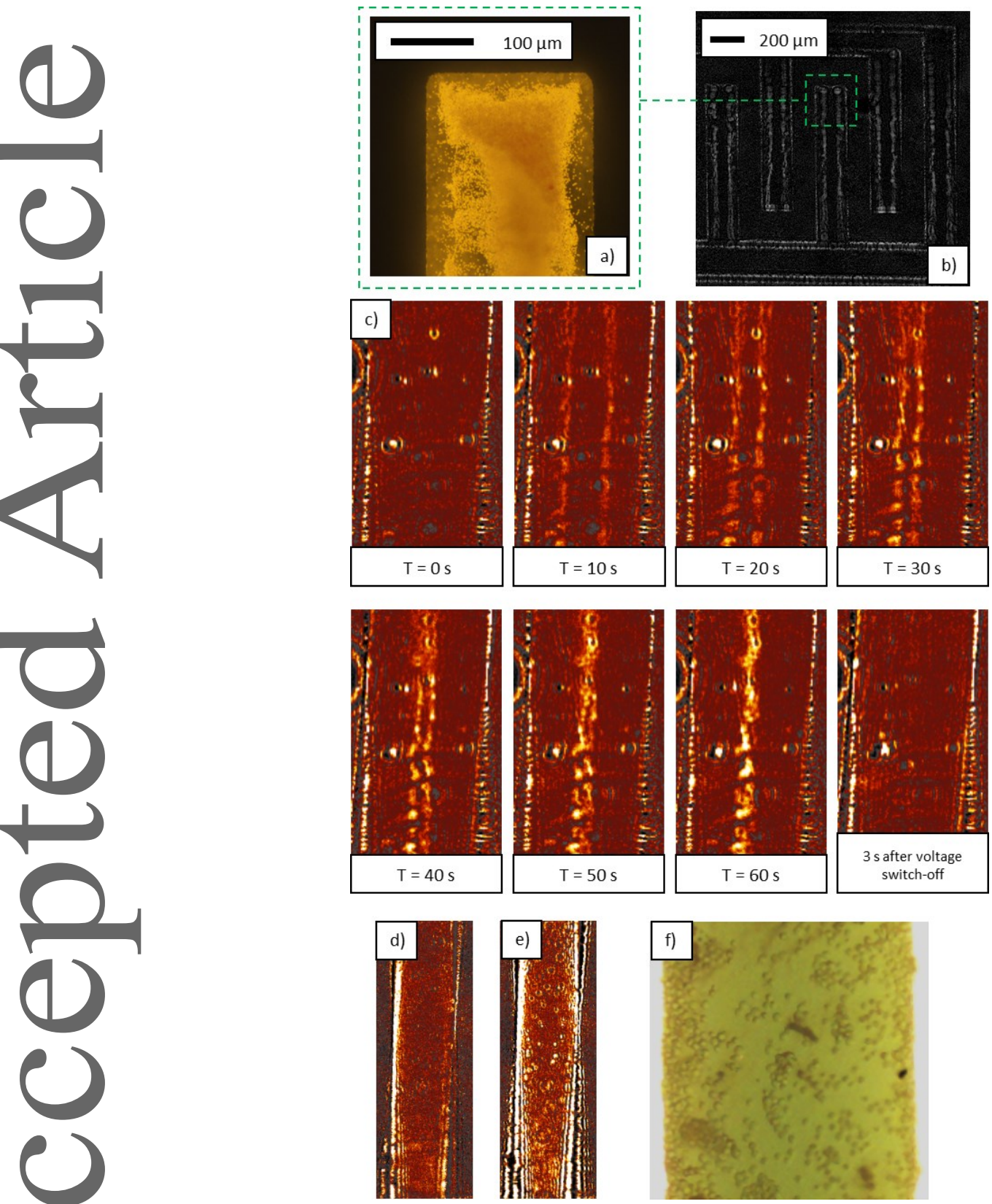

Figure 2 : Comparison of a) dark field optical microscopy imaging and b) SPRI observations of the collection of $1 \mu \mathrm{m}$ polystyrene beads on the electrodes. c) SPRI observation of the collection dynamics of $200 \mathrm{~nm}$ polystyrene beads on one electrode. $d$ ) and e) SPRI observation of the collection of yeasts $(5-10 \mu \mathrm{m})$ on one electrode before (d) and after (e) applying the voltage. f) Optical microscopy image of the yeasts trapped on the electrode. All of these trapping experiments have been repeated at least ten times and the same results have been observed each time. 
Figure 3

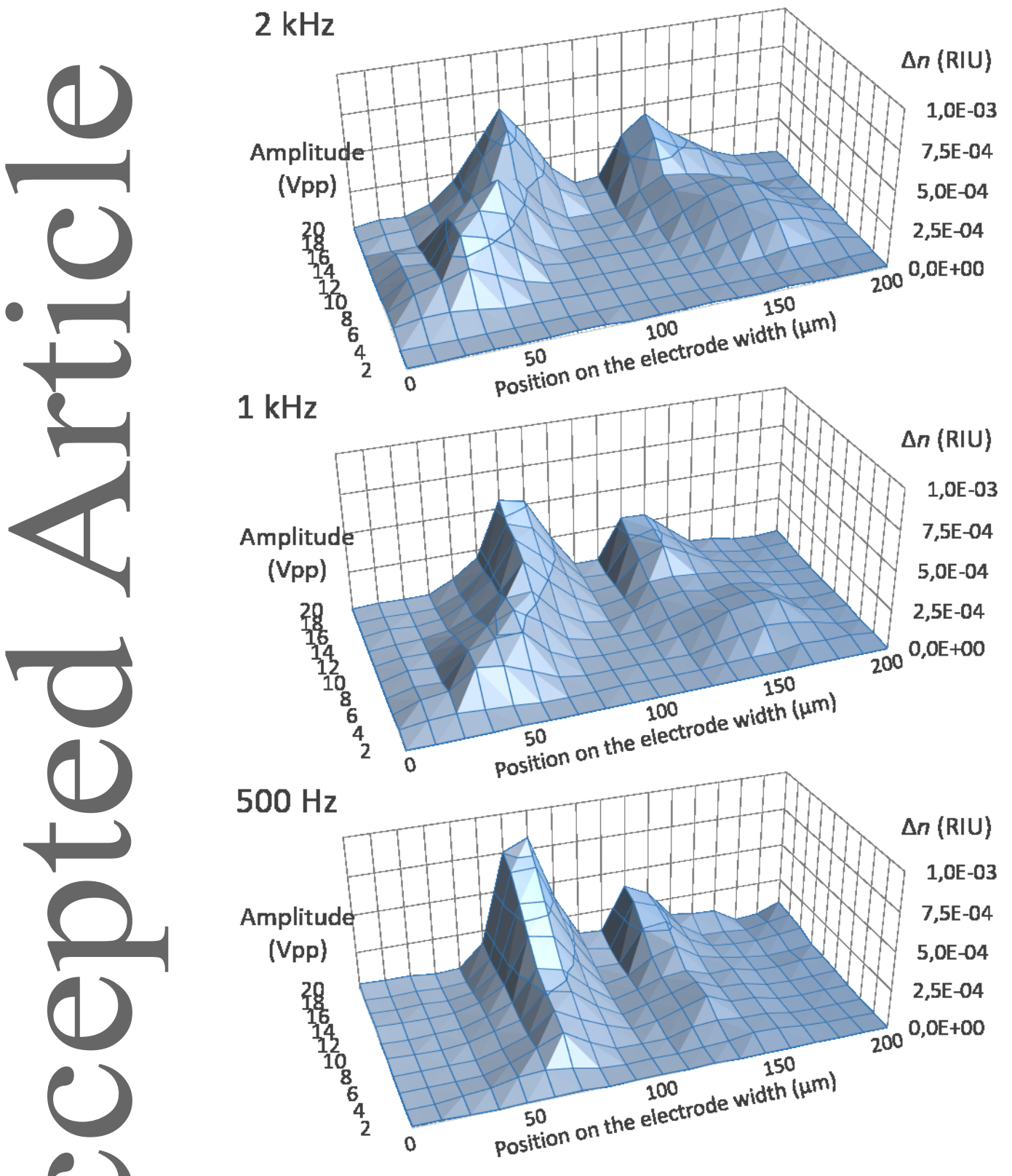

Figure 3: 3D representation of the trapped $200 \mathrm{~nm}$ nanobead position: optical index variations (vertical axis) are displayed over the electrode width (on the 0-200 $\mu \mathrm{m}$ horizontal axis) as a function of the electrical amplitude (on the 0-20 Vpp horizontal axis) for the 3 studied frequencies $(2 \mathrm{kHz}, 1 \mathrm{kHz}, 500 \mathrm{~Hz}) 30 \mathrm{~s}$ after beginning to apply the electric field. This experiment has been repeated twice in the same conditions but with a different chip (of identical geometry) and a new solution of beads each time. The results were qualitatively identical. 
Figure 4
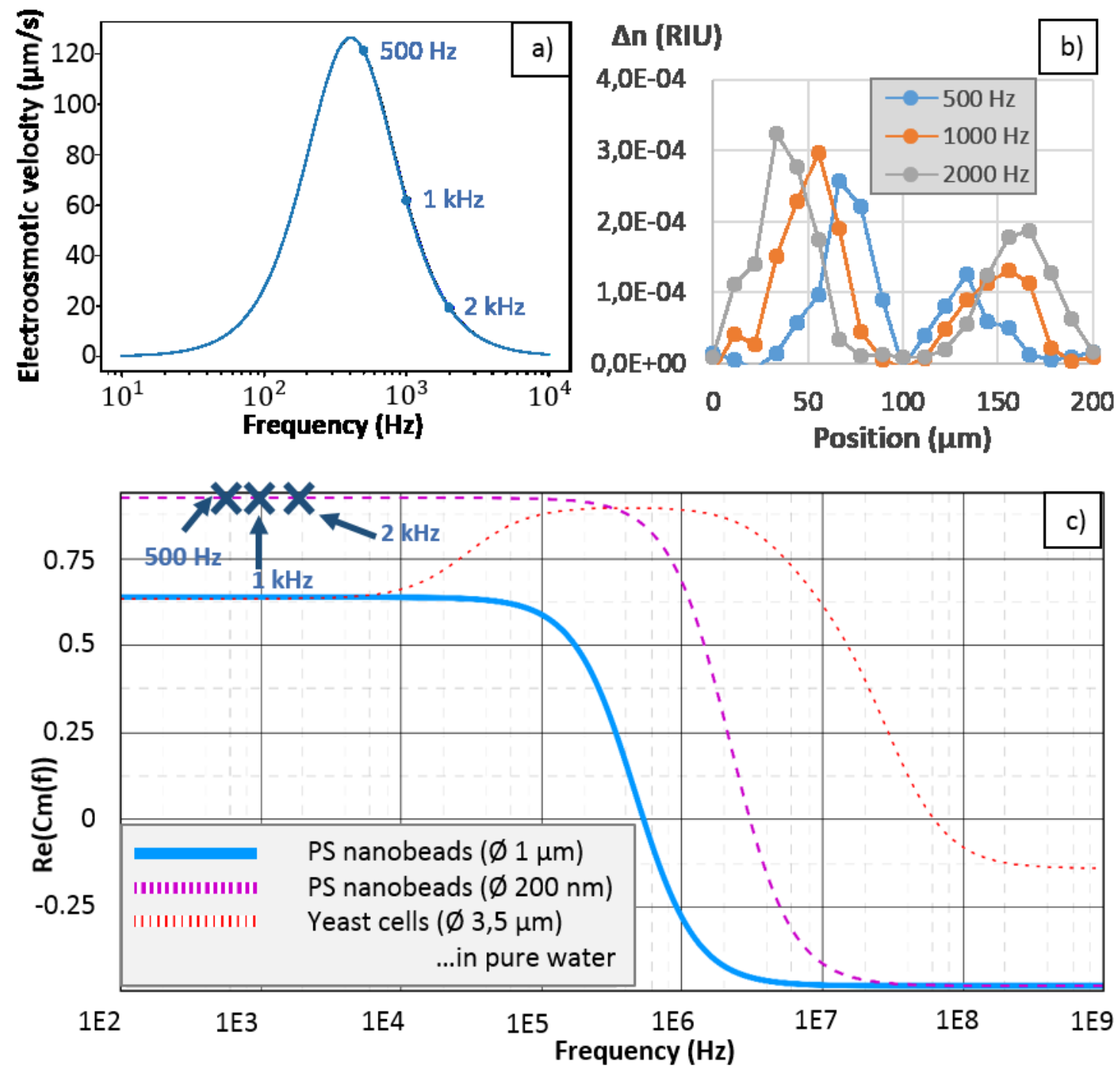

Figure $4:$ a) Electroosmotic velocity at the electrode surface at $50 \mu \mathrm{m}$ from the electrode edges. Calculations were made according to the theory explained in [25] b) Experimental collection positions of the beads along the electrode width after $30 \mathrm{~s}$, observed for 3 frequencies at $20 \mathrm{Vpp}$. c) Real part of the Clausius-Mossoti factor for $200 \mathrm{~nm}$ polystyrene beads in DI water. 
Figure 5

a)

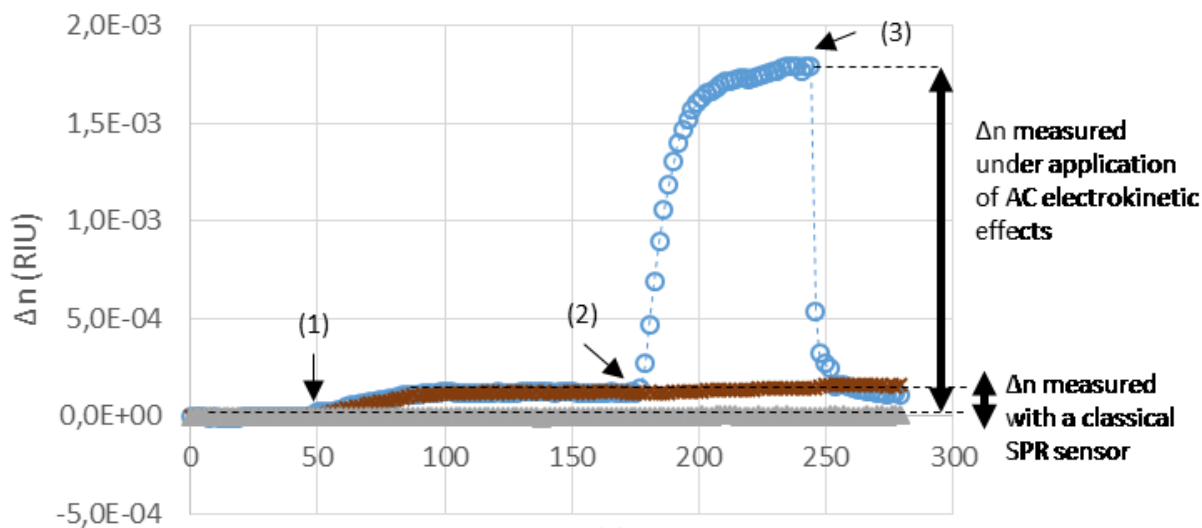

Time (s)

-.-.-- Microstructured sensor -.- $\mathbf{x}$-.- Classical SPR sensor

--- Negative control

TM/IE (\%)

1E-01

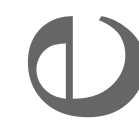

12

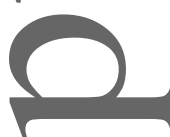

1E-03

( )

C

()

1E-04

1000

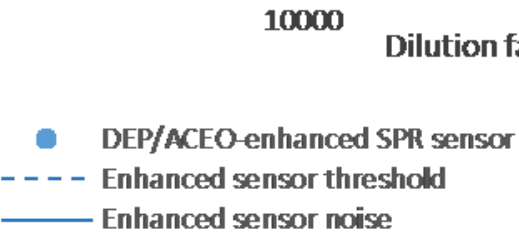

b)

\section{)}

- DEP/ACEO enhanced SPR sensor

Enhanced sensor noise
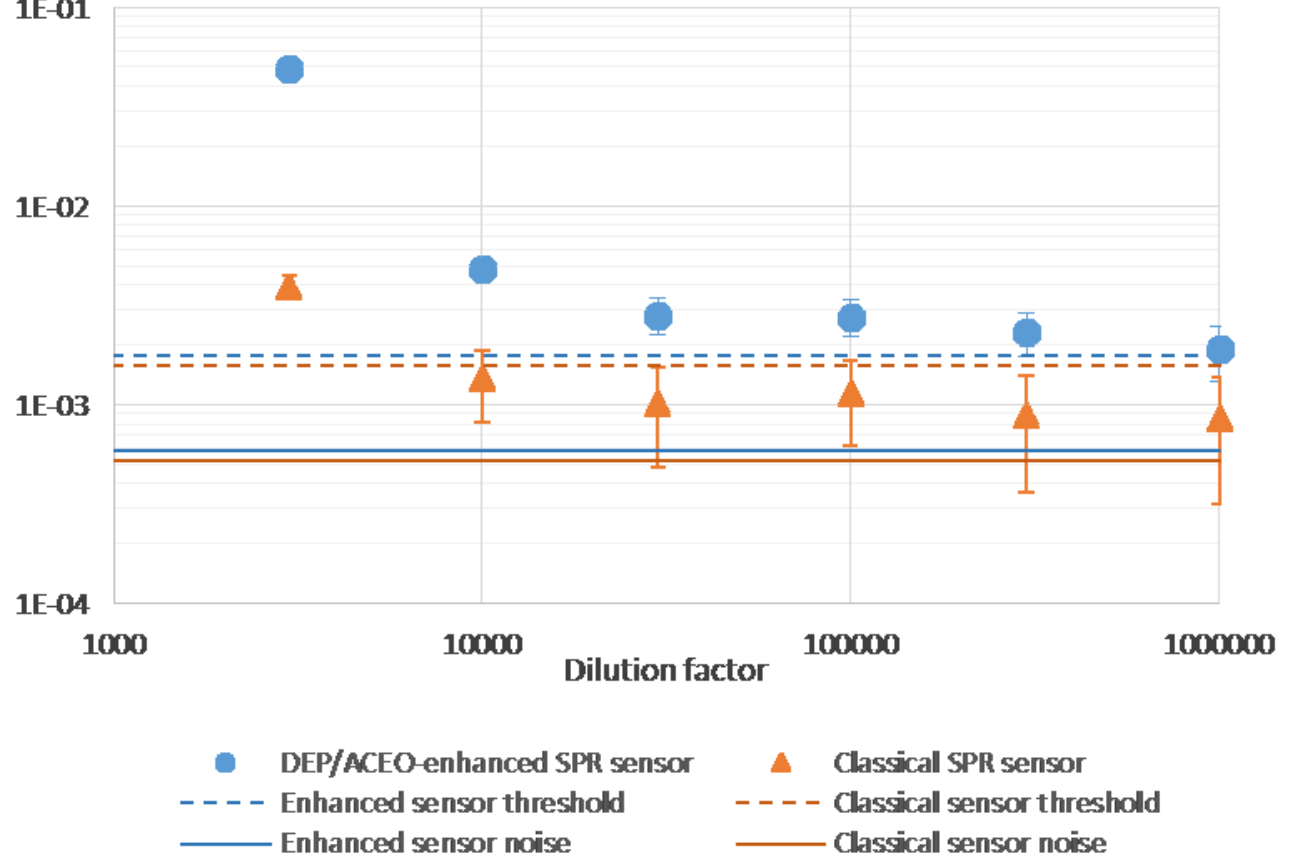

4 Classical SPR sensor

_ Classical sensor noise

This article is protected by copyright. All rights reserved. 
Figure $5:$ a) Example of kinetic monitoring of the signal variations during the injection of a solution of polystyrene beads $(200 \mathrm{~nm}$ ) with a 1000 dilution factor into our biosensor. Both curves were obtained on the same device, during the same experiment, at the same time. A sinusoidal voltage ( $2 \mathrm{kHz}, 20 \mathrm{Vpp}$ ) was applied on the electrodes. Steps sequence: (1) Beads are injected. (2) The function generator is switched on. (3) The generator is switched off. Negative control (signal measured while applying the same voltage on ultrapure water only) is plotted for comparison. b) Signal variations for various dilutions of the solution. Noise and detection threshold were calculated for both electrodes and displayed for convenience (error bars also represent the noise). This experiment has been repeated three times in the same conditions, with a different sample and new dilutions of beads each time. Observed results demonstrated the same enhancement of the limit of detection.

Supporting Information

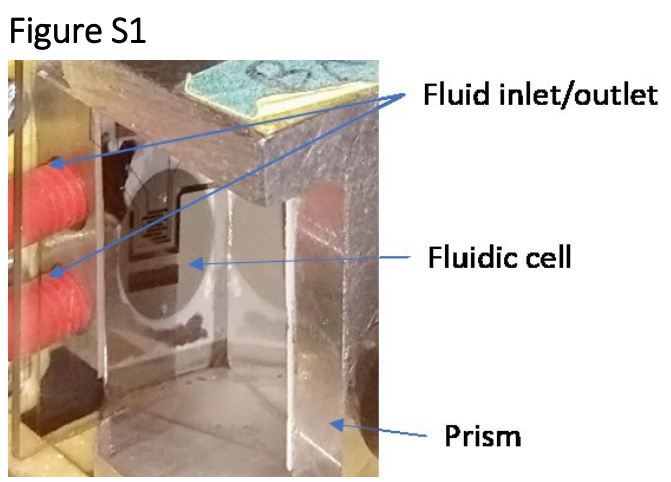

Figure S1: Picture of the fluidic cell.

\section{Figure S2}

\section{Signal \\ variations, \\ normalized}

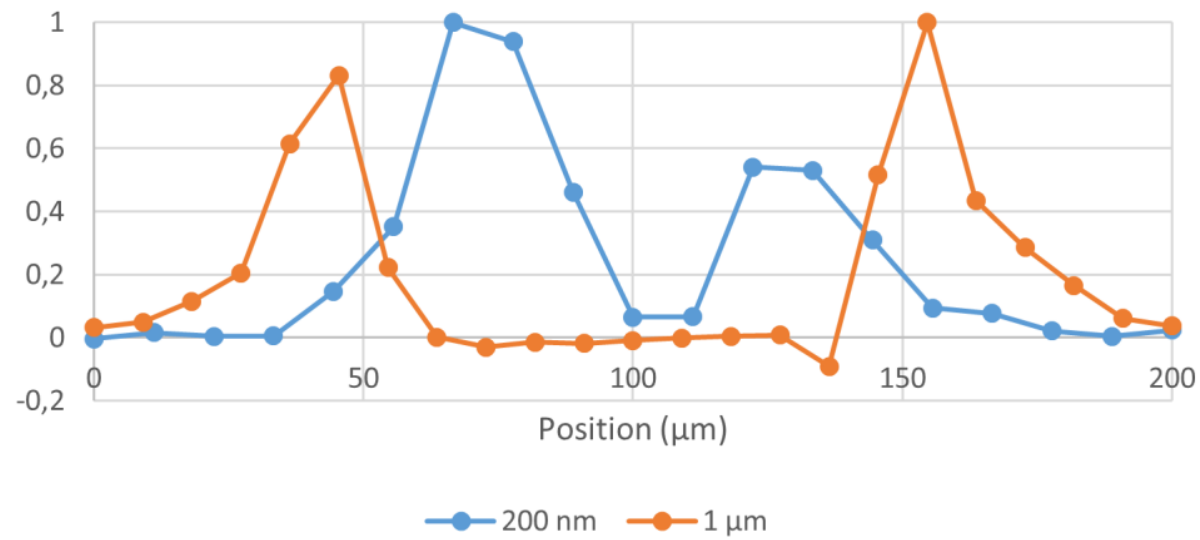

Figure S2: Experimental collection positions of the beads along the electrode width (one edge is at 0 $\mu \mathrm{m}$, the other one at $200 \mu \mathrm{m})$ when applying a sinusoidal voltage $(1 \mathrm{kHz}, 10 \mathrm{Vpp})$ during $30 \mathrm{~s}$, depending on the size of the bead solution. Signal variations are normalized to ensure comparability 
( $1 \mu \mathrm{m}$ beads induce much bigger variations than $200 \mathrm{~nm}$ beads). As explained in the discussion, the position of the $1 \mu \mathrm{m}$ beads is closer to the edge because the DEP force grows faster than ACEO with the size of the targeted object.

Parameters used in MyDEP for latex beads.

Parameters used in MyDEP calculations to study the dielectrophoretic behavior of beads have been obtained from a method adapted from pioneer work by Green and Morgan ([Green, N.G., Morgan, H. J. Phys. Chem. B 1999, 103, 41-50]) and later described in ([M .Suzuki,T. Yasukawa,Y. Mase,D. Oyamatsu, H. Shiku, and, and T. Matsue, Langmuir 200420 (25), 11005-11011]) and ([S. Menad, A. El-Gaddar, N. Haddour, S. Toru, M. Brun, F. Buret, and M. Frenea-Robin, Langmuir 201430 (19), 5686-5693]).

The effective conductivity of a latex particle can be expressed as:

$$
\sigma_{p}=\sigma_{p b u l k}+\frac{2 K_{s}^{i}}{r}+\frac{2 K_{s}^{d}}{r}
$$

Where $r$ is the particle radius, $\sigma_{\text {pbulk }}$ is the conductivity of latex and $K_{S}^{i}$ and $K_{S}^{d}$ are the Stern layer and the diffusion layer conductance, respectively. $\sigma_{p b u l k}$ is negligible, which is also the case of $K_{s}^{d}$ when the particles are immersed in a low conductivity medium such as DI water. The $\sigma_{p}$ value can be deduced from the experimental determination of the crossover frequency $f_{0}$ by considering that the real part of the Clausius-Mossotti factor $\operatorname{Re}[\mathrm{CM}]$ cancels at $f=f_{0}$.

As regards $200 \mathrm{~nm}$ amino beads, the $\sigma_{\mathrm{p}}$ value was found to be $18.7 \times 10^{-3} \mathrm{~s} / \mathrm{m}$ under the experimental conditions, $\varepsilon_{m}=80 \varepsilon_{0} \quad$ (farads/meter) and $\sigma_{m}=5 \times 10^{-4} \mathrm{~S} / \mathrm{m}$ and considering $\varepsilon_{p}=2.56 \varepsilon_{0}$ (farads/meter)

As regards $1 \mu \mathrm{m}$ amino beads, the $\sigma_{\mathrm{p}}$ value was found to be $3.15 \times 10^{-3} \mathrm{~S} / \mathrm{m}$ under similar conditions. 